\title{
ANÁLISE DO ACOMPANHAMENTO E FATORES DE RISCO PARA O ACIDENTE VASCULAR CEREBRAL EM HIPERTENSOS
}

\author{
Analysis of Monitoring and Risk Factors for Vascular Cerebral Accident in Hypertensions \\ Análisis del Acompañamiento y Factores de Riesgo para el Accidente Vascular Cerebral \\ en Hipertensos
}

Diego da Silva Bezerra ${ }^{1 *}$, Macerlane de Lira Silva ${ }^{1}$, Renata de Oliveira Freire Araújo ${ }^{1}$, Geane Silva Oliveira $^{1}$, José Maxwell de Araújo da Silva ${ }^{1}$, Ítala Maria Rosendo da Silva ${ }^{1}$, Symara Abrantes Albuquerque de Oliveira Cabral ${ }^{1}$, Cícera Kaline Gomes Barreto ${ }^{2}$, Ivson Vinícius Avelino Galindo ${ }^{2}$, Renata Lívia Silva Fonsêca Moreira de Medeiros ${ }^{1}$, Kaio Teixeira de Araújo ${ }^{1}$, Teógenes de Oliveira ${ }^{1}$, Ayli Micaelly da Silva ${ }^{1}$, Saullo de Alcântara Mendes², Joberlânia Mamede Carneiro Rodrigues'.

\section{RESUMO}

Objetivo: Analisar o acompanhamento e fatores de risco para o AVC em hipertensos, além de verificar a incidência de hipertensos, identificar os fatores que podem influenciar na adesão ao tratamento e averiguar os riscos cardiovasculares dos hipertensos. Métodos: Trata-se de uma pesquisa de campo, exploratória, descritiva, com abordagem quantitativa, realizada em uma Unidade Básica de Saúde de Cajazeiras, na Paraíba, sendo a população do estudo composta por hipertensos cadastrados no programa HIPERDIA. Resultados: Observou-se uma parcela significativa de portadores de HAS idosos, do sexo feminino, com pouca escolaridade, expostos a fatores de risco como o sobrepeso/obesidade, sedentários, em sua maioria com antecedentes familiares significativos, e ainda com fatores de risco associados, especialmente o diabetes mellitus. Observou-se uma autopercepção positiva das condições de saúde, entretanto os indivíduos estão sob tratamento medicamentoso intensivo, com uso de diversas drogas individualizadas e associadas, atrelado a realização precária de exames laboratoriais. Conclusão: Observou-se alto risco para AVC nos portadores de HAS avaliados, como o sedentarismo. Importante considerar que uma percepção positiva sobre a saúde pode influenciar no déficit de autocuidado pela pouca percepção dos riscos da doença à vida, o que demanda dos profissionais atuantes uma tomada de atitudes frente a tal realidade.

Palavras-chave: Acidente vascular cerebral, Fatores de riscos, Hipertensão.

\begin{abstract}
Objective: To analyze the follow-up and risk factors for stroke in hypertensive patients, as well as to verify the incidence of hypertension, to identify factors that may influence adherence to treatment, and to investigate the cardiovascular risks of hypertensive individuals. Methods: This is an exploratory, descriptive, quantitative study conducted at a Basic Health Unit in Cajazeiras, Paraíba, Brazil, the study population being composed of hypertensive patients enrolled in the HIPERDIA program. Results: There was a significant proportion of elderly women with low schooling who were exposed to risk factors such as overweight / obesity, sedentary individuals, mostly with a significant family history, and associated risk factors, especially diabetes mellitus. A positive self-perception of the health conditions was observed, although the individuals are under intensive drug treatment, using several individualized and associated drugs, linked to the precarious accomplishment of laboratory tests. Conclusion: A high risk for stroke was observed in patients with hypertension, such as sedentary lifestyle. It is important to consider that a positive perception about health can influence the deficit of self-care due to the low perception of the risks of the disease to life, which demands of the acting professionals an attitude towards this reality.
\end{abstract}

Key words: Stroke, Risk Factors, Hypertension.

${ }^{1}$ Faculdade Santa Maria, Cajazeiras - PB. *E-mail: diegobezerra2108@gmail.com

2 Universidade Federal de Campina Grande - UFCG. 


\section{RESUMEN}

Objetivo: Analizar el seguimiento y factores de riesgo para el AVC en hipertensos, además de verificar la incidencia de hipertensos, identificar los factores que pueden influir en la adhesión al tratamiento, y averiguar los riesgos cardiovasculares de los hipertensos. Metodologia: Se trata de una investigación de campo, exploratoria, descriptiva, con abordaje cuantitativo, realizada en una Unidad Básica de Salud de Cajazeiras, en Paraíba, siendo la población del estudio compuesta por hipertensos registrados en el programa HIPERDIA. Resultados: Se observó una porción significativa de portadores de HAS ancianos, del sexo femenino, con poca escolaridad, expuestos a factores de riesgo como el sobrepeso / obesidad, sedentarios, en su mayoría con antecedentes familiares significativos, y aún con factores de riesgo asociados, especialmente la diabetes mellitus. Se observó una autopercepción positiva de las condiciones de salud, sin embargo los individuos están bajo tratamiento medicamentoso intensivo, con uso de diversas drogas individualizadas y asociadas, vinculado a la realización precaria de exámenes de laboratorio. Conclusíon: Se observó un alto riesgo para AVC en los portadores de HAS evaluados, como el sedentarismo. Es importante considerar que una percepción positiva sobre la salud puede influir en el déficit de autocuidado por la poca percepción de los riesgos de la enfermedad a la vida, lo que demanda de los profesionales actuantes una toma de actitudes frente a tal realidad.

Palabras clave: Accidente vascular cerebral, Factores de riesgo, Hipertension.

\section{INTRODUÇÃO}

Nos últimos anos a pirâmide etária brasileira vem progressivamente mudando, especialmente com o processo de envelhecimento populacional frente a maior expectativa de vida. Há ainda um novo perfil epidemiológico, de modo que a morbimortalidade por doenças infectocontagiosas vai dando espaço para as Doenças Crônicas Não Transmissíveis (DCNT) (SOUZA MFM, et al., 2018; SILVA JB, et al., 2014).

De acordo com Azevedo ECC et al. (2014) as DCNT constituem-se como as principais causas de mortes no mundo, em decorrência, especialmente, de inadequados hábitos alimentares e de vida, como o sedentarismo.

Dentre as DCNT a Hipertensão Arterial Sistêmica (HAS) é uma das mais prevalentes, com uma estimativa global de 37,8\% de acometimento em homens e 31\% de mulheres, e no Brasil com uma prevalência acima de $30 \%$. Constitui-se de uma doença multifatorial que se apresenta como de alta prevalência e difícil controle, sendo considerada dentre os principais problemas de saúde pública, especialmente pelo fator de risco associado às Doenças Cardiovasculares (DCV) com complicações associadas aos sistemas renal, encefálico e vascular (MACHADO JC et al. 2016).

De acordo com Silva JB, et al. (2014), cerca de metade da população acima dos 50 anos de idade é portadora de HAS, e esse aumento na prevalência está associado, de acordo com Muraro AP, et al. (2013) a vários fatores, dentre eles: sedentarismo, estresse, tabagismo, envelhecimento, gênero, aumento do peso corpóreo e dieta.

Tem-se, na HAS uma pressão arterial com persistência acima de 135mmHg para sistólica e $85 \mathrm{mmHg}$ para diastólica (MOTTER RF, et al. 2013). São estabelecidos pelas VI Diretrizes de Monitorização Ambulatorial da Pressão Arterial e IV Diretrizes de Monitorização Residencial da Pressão Arterial da Sociedade Brasileira de Cardiologia (NOBRE F, et al., 2018) as seguintes caracterizações e medidas: normotensão verdadeira (< $140 / 90 \mathrm{mmHg}$ e na MAPA de 24 horas $<130 / 80 \mathrm{mmHg}$, ou na MRPA $<135 / 85 \mathrm{mmHg}$ ); hipertensão verdadeira $(\geq 140 / 90 \mathrm{mmHg}$ e médias igualmente anormais de 24 horas pela MAPA $\geq 130 / 80 \mathrm{mmHg}$, ou pela MRPA $\geq$ $135 / 85 \mathrm{mHg}$ ); efeito do avental branco (diferença entre a medida da PA no consultório e a média da MAPA na vigília ou da MRPA $\geq 135 / 85 \mathrm{mmHg}$, quando superior a 20 e $10 \mathrm{mmHg}$, respectivamente, nas pressões sistólica e diastólica).

Quanto ao controle e tratamento, Motter RF, et al. (2013) aborda inicialmente o não farmacológico, pautado na adoção de estilo de vida saudável e, nos casos de persistência, o farmacológico pelo uso de medicamentos associado ao anterior. 
Quando a adoção de hábitos saudáveis Jodas EMMG, et al. (2014) aponta como estratégias: redução da ingestão de sódio, aumento na ingesta de potássio e magnésio, adoção de práticas de atividades físicas aeróbicas, redução do peso corpóreo e diminuição do estresse.

Importante considerar que as principais consequências do aumento da pressão arterial são: o AVC e o Infarto Agudo do Miocárdio (IAM) (GUERRA DR, et al., 2016). Com base nos dados epidemiológicos, Bensenor IM, et al. (2015) apontam que a Pesquisa Nacional de Saúde (PNS), a partir de inquérito com base domiciliar, estima uma população de 2.232 .000 pessoas com AVC e 568.000 portadoras de incapacidades graves por AVC, com uma prevalência de 1,6\% em homens e 1,4\% em mulheres, e a de incapacidade $29,5 \%$ em homens e de $21,5 \%$ em mulheres, havendo ainda uma progressão na prevalência a partir do aumento da idade e redução dos níveis de escolaridade.

De acordo com Guerra DR, et al. (2016) o AVC acontece a partir da completa interrupção do fluxo sanguíneo cerebral, seja pelo rompimento de um vaso encefálico no tipo isquêmico, ou pelo rompimento de um vaso do encéfalo no tipo hemorrágico. Sendo que o mais frequente é o do tipo isquêmico ( $80 \%)$ em comparação ao hemorrágico (15\%).

As experiências vivenciadas nas ações de saúde evidenciaram, em diversos estudos, a efetividade no sentido de fortalecer as ações de prevenção aos agravos, de modo que, em 1994, o Brasil vivenciou a implantação do programa Saúde da Família (PSF), posteriormente elevado à Estratégia Saúde da Família, que tem por objetivo a organização das ações de saúde à nível de promoção, proteção e cuidados à saúde, ampliando a atenção e o acesso, conforme especificam Carrapato JFL, et al. (2017).

Em consonância a tal mudança, o Ministério da Saúde, através da Portaria 235/GM de 22/02/2001, estabeleceu diretrizes para a reorganização da atenção aos segmentos populacionais expostos e portadores de hipertensão arterial e de diabetes mellitus.

Esse documento subsidiou a elaboração do Plano de Reorganização da Atenção à HAS e ao Diabetes Mellitus (DM), desencadeando na construção, em 2002, do HIPERDIA Sistema de Cadastramento e Acompanhamento de Hipertensos e Diabéticos (BRASIL MS, 2006).

Em 04 de março de 2002 foi elaborada a portaria no 371/GM para o Programa Nacional de Assistência Farmacêutica para HA e o DM, que tratava da organização, assistência, prevenção, promoção à saúde, vinculação dos usuários à rede básica de saúde do SUS, bem como da implementação de programas de educação permanente para essas doenças e para os demais fatores de risco para as doenças cardiovasculares (PAULA PAB, et al., 2011).

Segundo Brasil MS (2002) o Programa estabelece responsabilidades solidárias, inclusive financiamento, entre União, Estados e Municípios, com objetivos de colocar o cadastramento dos portadores de hipertensão e diabetes mediante a instituição do cadastro nacional dos portadores dessas patologias; ofertar de maneira contínua para rede básica os medicamentos para hipertensão (hidroclorotiazida $25 \mathrm{mg}$, propranolol $40 \mathrm{mg}$, captopril $25 \mathrm{mg}$ ) e diabetes (glibenclamida $5 \mathrm{mg}$, metformina $850 \mathrm{mg}$ e insulina); acompanhar e analisar os impactos na morbimortalidade para estas doenças, decorrentes da implementação do Programa Nacional .

Desse modo, o trabalho que se segue busca analisar os fatores que se sobressaem em uma população usuária da atenção básica e o programa HIPERDIA, com o intuito de embasar a comunidade científica e profissionais de saúde a respeito de fatores controláveis e contribuintes para ocorrência do AVC, possibilitando a detecção precoce do quadro clínico cardiovascular desfavorável que poderá acometer a população e onerar o sistema público de saúde com gastos na atenção terciária, o qual pode ser evitável por meio de medidas preventivas e promoção da saúde ainda na atenção básica.

Assim, o presente trabalho tem por objetivo geral analisar o acompanhamento e fatores de risco para 0 AVC em hipertensos, e como objetivos específicos: verificar a incidência de hipertensos; identificar os fatores que podem influenciar a na adesão ao tratamento; e averiguar os riscos cardiovasculares dos hipertensos a partir do acompanhamento, partindo da hipótese de que o acompanhamento dos hipertensos pela atenção básica não é realizado de forma sistemática. 


\section{MÉTODOS}

Trata-se de uma pesquisa de campo, exploratória, do tipo descritiva, prospectivo e analítico, com abordagem quantitativa, realizada na UBS Família, localizada no bairro Capoeiras, no município de Cajazeiras, distante $477 \mathrm{~km}$ da capital João Pessoa, com uma população de 61.816 habitantes e uma área territorial de $565.899 \mathrm{~km}^{2}$.

A população do estudo foi composta por 250 pacientes hipertensos cadastrados no programa HIPERDIA da referida UBS, já a amostra, a partir do critério de inclusão (pacientes hipertensos cadastrados no programa do HIPERDIA), foi estatisticamente definida em 41 participantes.

O instrumento para coleta de dados da pesquisa foi um questionário constituído a partir da ficha de acompanhamento do hipertenso e diabético do HIPERDIA, composto por quatro partes, sendo a primeira contemplando questões sociodemográficas, para caracterização das participantes, a segunda referente aos antecedentes pessoais, à terceira sobre os dados clínicos do paciente e a quarta os dados relativos aos medicamentos.

Foram estabelecidos por critérios de inclusão: hipertensos acompanhados e cadastrados no Programa; aqueles que dispuseram a participar da pesquisa, e como critérios de exclusão: os que estavam cadastrados, mas sem acompanhamento periódico, bem como os inicialmente acompanhados sem cadastramento, e ainda os que se recusaram a participarem da pesquisa.

A coleta de dados foi realizada após aprovação do projeto Comitê de Ética e Pesquisa (CEP) da Faculdade Santa Maria, sob registro 82800218.0.0000.5180, obedecendo às diretrizes e às normas éticas determinadas na Resolução 466/12 do Conselho Nacional de Saúde (CNS) (BRASIL MS, 2013).

As entrevistas foram realizadas no período de maio de 2018 a agosto de 2018, prosseguindo com a análise de dados, categorizados no Microsoft Office Excel, e posteriormente registrados em forma de banco de dados no programa Statistical Package for the Social Sciences (SPSS), versão 21.0, e analisados por meio da descrição das variáveis.

\section{RESULTADOS E DISCUSSÃO}

Verificou-se que a idade média dos participantes foi de 61,3 (DP $\pm 10,6)$. Em relação ao gênero, prevaleceu o feminino com $78 \%$, no estado civil foi identificada uma predominância no status casado (a) com $51,2 \%$. Ademais, no que concerne a escolaridade, o ensino fundamental I incompleto teve maior incidência (41,5\%), já a profissão verificada com mais dominância foi a de aposentado (a) com 48,8\%, em sua maioria motivada por a idade (65\%).

Estudo realizado por Firmo JOA, et al. (2018), que abrangeu uma população de 9.412, encontrou média de idade de 64,9 anos, desses $60,2 \%$ eram mulheres e $37 \%$ possuíam menos de 4 anos de estudo, corroborando, assim, com os dados encontrados.

Nesse sentido, importante se faz considerar que há uma proporcionalidade entre escolaridade e conhecimentos acerca da prevenção, de modo que tal processo tem menos adesão quanto menor a escolaridade (SILVEIRA EL, et al., 2018, p.171). Já estudo de Oliveira PTGO e Almeida JM (2018, p.147) demonstra dificuldade na manutenção terapêutica à medida que se amplia a complexidade da situação, tornando o tratamento cada vez mais complexo em decorrência de menor grau de escolarização.

Na variável jornada de trabalho, o período diurno teve a maior ocorrência $(100 \%)$, no que tange a carga horária diária, esta apresentou média de 9,3 horas/dia $(\mathrm{DP} \pm 1,988)$ e o tempo de ocupação desta função laboral teve média de 29,7 anos ( $\mathrm{DP} \pm 13,249)$. A jornada de trabalho pode influir negativamente no estresse, que, por sua vez constitui-se como um fator de risco para a HAS (SILVA JP, et al., 2014).

Os dados foram dispostos em tabelas, à priori, contendo as variáveis investigadas, sua frequência, porcentagem, medida de tendência central (média) e desvio padrão (DP). 
Partindo dessas considerações, a Tabela 1 vem apresentar os dados sociodemográficos que caracterizam a amostra selecionada para compor a pesquisa.

Tabela 1 - Dados sociodemográficos de portadores de HAS cadastrados no HIPERDIA. Cajazeiras - PB.

\begin{tabular}{|c|c|c|c|c|}
\hline Variáveis & Frequência & $\begin{array}{l}\text { Porcentagem } \\
(\%)\end{array}$ & Média & $\begin{array}{c}\text { Desvio } \\
\text { Padrão (DP) } \\
\end{array}$ \\
\hline \multicolumn{5}{|l|}{ Gênero } \\
\hline Masculino & 9 & $22 \%$ & - & - \\
\hline Feminino & 32 & $78 \%$ & & \\
\hline \multicolumn{5}{|l|}{ Estado civil } \\
\hline Solteiro (a) & 6 & $14,6 \%$ & & \\
\hline Casado (a) & 21 & $51,2 \%$ & & \\
\hline Separado (a) & 2 & $4,9 \%$ & - & - \\
\hline Viúvo (a) & 10 & $24,4 \%$ & & \\
\hline Outros & 2 & $4,9 \%$ & & \\
\hline \multicolumn{5}{|l|}{ Escolaridade } \\
\hline Ensino fundamental I completo & 2 & $4,9 \%$ & & \\
\hline Ensino fundamental II completo & 2 & $4,9 \%$ & & \\
\hline Ensino médio completo & 5 & $12,2 \%$ & & \\
\hline Ensino fundamental I incompleto & 17 & $41,5 \%$ & - & - \\
\hline Ensino fundamental II incompleto & 1 & $2,4 \%$ & & \\
\hline Ensino médio incompleto & 1 & $2,4 \%$ & & \\
\hline \multirow{2}{*}{\multicolumn{5}{|c|}{ Profissão }} \\
\hline & & & & \\
\hline Agricultor (a) & 8 & $19,5 \%$ & & \\
\hline Aposentado (a) & 20 & $48,8 \%$ & & \\
\hline Do lar & 5 & $12,2 \%$ & - & - \\
\hline Doméstica & 3 & $7,3 \%$ & & \\
\hline Outros & 4 & $9,8 \%$ & & \\
\hline Omissos & 1 & $2,4 \%$ & & \\
\hline \multicolumn{5}{|l|}{ Aposentado por? } \\
\hline Por idade & 13 & $65 \%$ & & \\
\hline Agricultor (a) & 1 & $5 \%$ & & \\
\hline Auxílio doença & 4 & $20 \%$ & - & - \\
\hline Pensionista & 1 & $5 \%$ & & \\
\hline Tempo de serviço & 1 & $5 \%$ & & \\
\hline \multicolumn{5}{|l|}{ Jornada de trabalho } \\
\hline Diurna & 17 & $100 \%$ & - & - \\
\hline Noturna & 0 & $0 \%$ & & \\
\hline Horas por dia & - & - & 9,3 & $\pm 1,988$ \\
\hline Tempo de trabalho na função? & - & - & 29,7 & $\pm 13,249$ \\
\hline
\end{tabular}

Fonte: Dados da pesquisa, 2018.

Quando questionados (as) sobre seus hábitos, 22 participantes (53,7\%) afirmaram considerar-se saudáveis, em contrapartida, 26 destes $(63,4 \%)$ relataram não praticar atividades físicas. No entanto, em relação à prática de atividade física, está fixou-se em $3 x$ ou mais por semana $(73,4 \%)$.

Estudos desenvolvidos por Dutra DD, et al. (2016) revelam que as doenças coronarianas têm por fatores de risco as circunferências abdominais aumentadas e a elevação do índice de Massa Corpórea, que, por sua vez, podem ser induzidos e/ou mantidos pelo sedentarismo.

Estudo conduzido por Hortencio MNS, et al. (2018) que avaliou os efeitos de exercícios físicos sobre fatores de risco cardiovascular em idosos hipertensos, em uma avaliação após três meses, concluiu que há eficácia na redução dos fatores de risco cardiovascular dos participantes, nesse caso, idosos sedentários, em sua maioria $(74 \%)$ do sexo feminino. 
Frente aos hábitos alimentares, 34 dos participantes $(82,9 \%)$ relataram possuir bons hábitos alimentares, no entanto, 31 dos sujeitos da amostra $(75,6 \%)$ disseram que costumam substituir as principais refeições por lanches. No que tange o estresse, 23 respondentes $(56,1 \%)$ consideram-se pessoas estressadas, dentre estes indivíduos participantes, 32 (78\%) afirmaram vivenciar episódios de estresse no contexto laboral. No mais, ao serem questionados sobre o uso de tabaco e de álcool, 38 dos participantes $(92,7 \%)$ verbalizaram que não fazem uso destas substâncias, respectivamente.

Entendendo que a HAS ocorre atrelada a fatores condicionantes e determinantes, dentre eles os proximais e os mais distais, ou seja, aqueles que não podem ser mudados e aqueles que podem ser modificados. Dentre aqueles que podem ser modificados tem-se os hábitos de vida, conforme a tabela 2.

Tabela 2 - Autopercepção de saúde e hábitos de vida de portadores de HAS cadastrados no HIPERDIA. Cajazeiras - PB.

\begin{tabular}{|c|c|c|c|c|}
\hline Variáveis & Frequência & $\begin{array}{l}\text { Porcentagem } \\
\text { (\%) }\end{array}$ & Média & Desvio Padrão (DP) \\
\hline \multicolumn{5}{|l|}{ Se considera saudável? } \\
\hline Sim & 22 & $53,7 \%$ & - & - \\
\hline Não & 19 & $46,3 \%$ & & \\
\hline \multicolumn{5}{|l|}{ Pratica atividade física? } \\
\hline Sim & 15 & $36,6 \%$ & - & - \\
\hline Não & 26 & $63,4 \%$ & & \\
\hline \multicolumn{5}{|l|}{ Frequência } \\
\hline $1 \times$ por semana & 2 & $13,3 \%$ & & \\
\hline $2 x$ por semana & 2 & $13,3 \%$ & - & - \\
\hline $3 x$ ou mais por semana & 11 & $73,4 \%$ & & \\
\hline \multicolumn{5}{|c|}{ Possui bons hábitos alimentares? } \\
\hline Sim & 34 & $82,9 \%$ & - & - \\
\hline Não & 7 & $17,1 \%$ & & \\
\hline \multicolumn{5}{|c|}{ Substitui as principais refeições? } \\
\hline $\operatorname{Sim}$ & 10 & $24,4 \%$ & - & - \\
\hline Não & 31 & $75,6 \%$ & & \\
\hline \multicolumn{5}{|l|}{$\begin{array}{l}\text { Se considera uma pessoa } \\
\text { estressada? }\end{array}$} \\
\hline $\operatorname{Sim}$ & 23 & $56,1 \%$ & - & - \\
\hline Não & 18 & $43,9 \%$ & & \\
\hline \multicolumn{5}{|c|}{$\begin{array}{l}\text { Costuma passar por situações } \\
\text { estressantes no trabalho? }\end{array}$} \\
\hline Sim & 9 & $22 \%$ & - & - \\
\hline Não & 32 & $78 \%$ & & \\
\hline \multicolumn{5}{|l|}{ Faz uso de tabaco? } \\
\hline Sim & 3 & $7,3 \%$ & - & - \\
\hline Não & 38 & $92,7 \%$ & & \\
\hline Quantos cigarros por dia? & & $100 \%$ & 3 & - \\
\hline \multicolumn{5}{|l|}{ Ingere bebida alcóolica? } \\
\hline Sim & 3 & $7,3 \%$ & - & - \\
\hline Não & 38 & $92,7 \%$ & & \\
\hline
\end{tabular}

Fonte: Dados da pesquisa, 2018.

Observa-se que os sujeitos da pesquisa possuem hábitos saudáveis no sentido do uso de substâncias, em contrapartida, observa-se uma autopercepção de eventos estressantes, entendendo que tal fato não pode ser modificado na vida de alguns deles. 
Tem-se ainda a percepção do autocuidado pelo não uso de substâncias tóxicas, como o cigarro e o álcool, que conforme Bezerra ALA, et al. (2019) configuram-se como fatores de risco para a HAS e suas complicações.

A Tabela 3 vem em complementariedade dos dados, apresentando informações pertinentes e referentes aos dados antropométricos. Sabe-se, ainda, que o déficit de tratamento, ou mesmo a potencialidade dos fatores de risco condicionam o desencadear de complicações que contribuem significativamente para o risco de mortalidade, as complicações relatadas pelos participantes também são descritas.

Tabela 3 - Dados antropométricos e complicações de portadores de HAS cadastrados no HIPERDIA. Cajazeiras - PB.

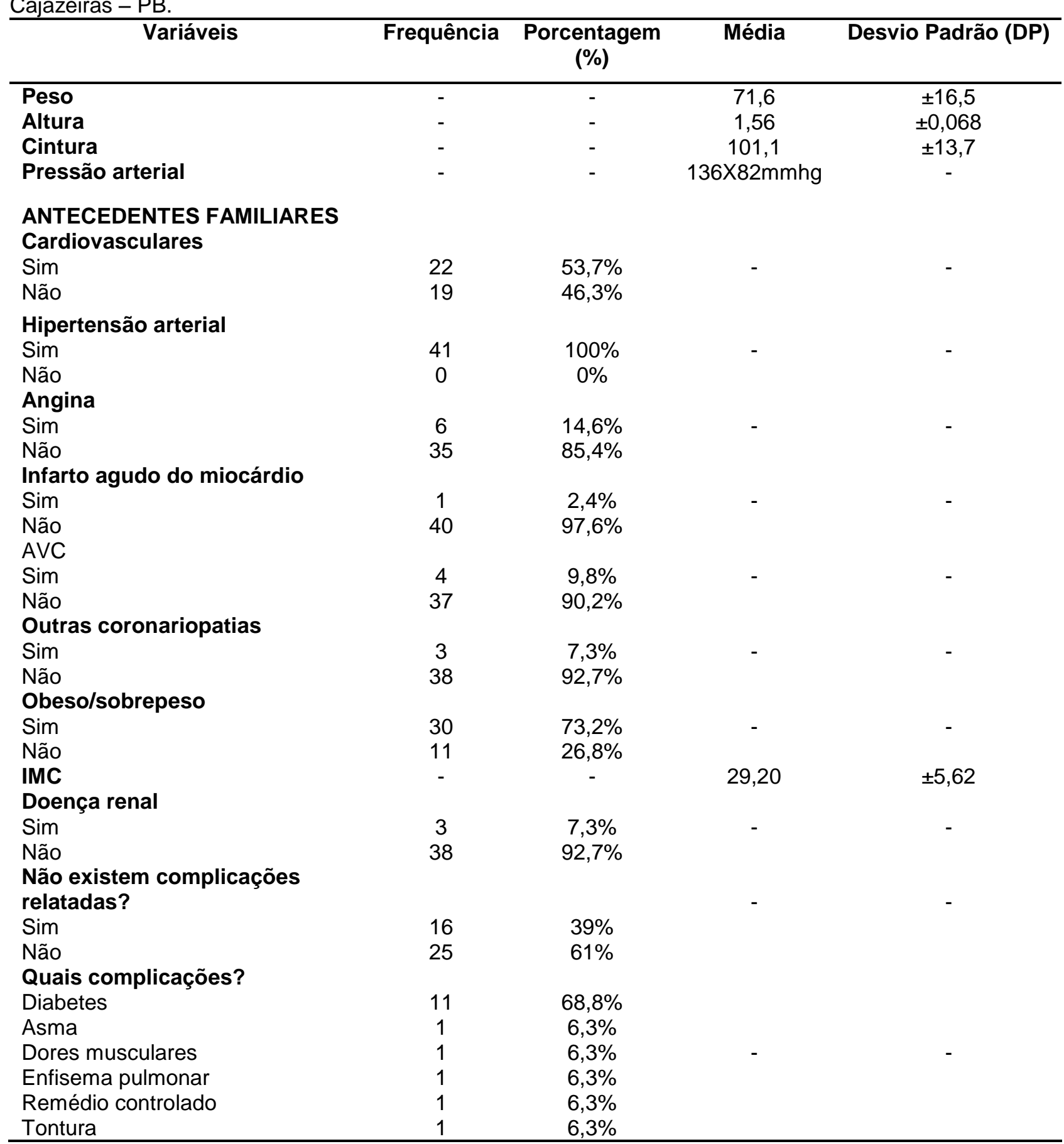

Fonte: Dados da pesquisa, 2018. 
Deste modo, foi observado que o peso dos participantes teve média de 71,6 ( $\mathrm{DP} \pm 16,5)$, a altura média foi de 1,56 (DP $\pm 0,068)$ e a cintura teve média de 101,1 (DP $\pm 13,7)$. Em relação aos antecedentes, $22(53,7 \%)$ destes afirmaram ter antecedentes cardiovasculares na família, $41(100 \%)$ relataram ter histórico familiar de hipertensão arterial, enquanto $35(85,4 \%)$ reportaram que não apresentam antecedentes de angina na família e $40(97,6 \%)$ não apresentam histórico de infarto agudo do miocárdio. Sobre o AVC e outras coronariopatias, $37(90,2 \%)$ e $38(92,7 \%)$ dos participantes afirmaram não apresentar nenhum antecedente familiar destes, respectivamente.

Dados sobre o peso e demais enfermidades também foram indagados, e, frente a isso, 30 dos sujeitos $(73,2 \%)$ componentes da amostra apresentaram indícios de estar acima do peso, apresentando IMC com média de 29,20 ( $D P \pm 5,62$ ). Revisão integrativa realizada por Bezerra ALA, et al. (2019) elenca como principais fatores de risco associados a HAS a alta circunferência abdominal, o excesso de peso, o consumo exacerbado de gorduras saturadas, os hábitos do tabagismo e etilismo, e ainda a realização irregular ou não realização de exercícios.

Dados da tabela 4 revelam ainda que, no que tange a doença renal, $38(92,7 \%)$ desses indivíduos afirmaram que não possuem tais antecedentes, resposta que assemelha-se a da presença de outras complicações, que teve 25 respostas (61\%) que negam a presença de complicações adicionais, no enquanto, das complicações citadas, diabetes foi a que apresentou maior prevalência, tendo 11 respostas $(68,8 \%)$.

De acordo com a VII Diretriz Brasileira de Hipertensão Arterial (MALACHIAS AR, et al., 2016) a associação entre HA e Diabetes Mellitus é capaz de dobrar o risco cardiovascular, e ainda, estando os fatores de risco para ambas as patologias atrelados como o sobrepeso, a obesidade, dentre outros.

Tecendo acerca do tratamento medicamentoso, tem-se na tabela 4 um panorama das medicações utilizadas pelos participantes e analisadas mediante o instrumento.

Foi verificada a administração de 22 tipos de medicações por parte dos participantes da pesquisa, frente ao tratamento de hipertensão arterial e diabetes mellitus, havendo prevalência do Hidroclorotiazida $(43,8 \%)$, Metformida (31,6\%), Losartana (39\%) e Captopril (17\%). No mais, as informações ampliadas acerca dessa variável estão descritas de modo detalhado na Tabela 4 , contento dados sobre as $\mathrm{mg}$, a quantidade de comprimidos ingeridos e a quantidade de administrações realizadas pelos pacientes ao longo do dia.

Tabela 4 - Medicamentos, mg, quantidade de comprimidos ingeridos e o número de vezes que a medicação foi administrada por dia a portadores de HAS cadastrados no HIPERDIA. Cajazeiras - PB.

\begin{tabular}{|c|c|c|c|c|}
\hline Variáveis (Medicamentos) & Frequência & Porcentagem(\%) & Média & Desvio Padrão (DP) \\
\hline Hidroclorotiazida $25 \mathrm{mg}$ & & & - & $\pm 1,263$ \\
\hline $1 / 2 \mathrm{cp}$ & 1 & $2,4 \%$ & & \\
\hline $1 \mathrm{cp}$ & 12 & $29,3 \%$ & & \\
\hline $1 \mathrm{cp} 1 / 2$ & 1 & $2,4 \%$ & & \\
\hline $2 \mathrm{cp}$ & 2 & $4,9 \%$ & & \\
\hline 2 cp $1 / 2$ & 1 & $2,4 \%$ & & \\
\hline Hidroclorotiazida $50 \mathrm{mg}$ & & & - & - \\
\hline $1 \mathrm{cp}$ & 1 & $2,4 \%$ & & \\
\hline Metformina & & & & $\pm 0,00$ \\
\hline 1x/dia & 4 & $9,8 \%$ & & \\
\hline Metformina 25mg & & & & - \\
\hline 1x/dia & 1 & $2,4 \%$ & & \\
\hline Metformina $50 \mathrm{mg}$ & & & & - \\
\hline 1x/dia & 1 & $2,4 \%$ & & \\
\hline Metformina 500mg & & & - & - \\
\hline 1x/dia & 1 & $2,4 \%$ & & \\
\hline Metformina $850 \mathrm{mg}$ & & & & $\pm 0,7527$ \\
\hline 1x/dia & 2 & $4,9 \%$ & & \\
\hline $2 x / d i a$ & 3 & $7,3 \%$ & & \\
\hline $3 x /$ dia & 1 & $2,4 \%$ & & \\
\hline
\end{tabular}




\begin{tabular}{|c|c|c|c|c|}
\hline \multirow{3}{*}{$\begin{array}{l}\text { Glibenglamida } \\
\text { Glibenglamida } 5 \mathrm{mg} \\
\text { 2x/dia } \\
\text { Enalapril } 10 \mathrm{mg}\end{array}$} & 1 & $2,4 \%$ & & \\
\hline & 1 & $2,4 \%$ & - & - \\
\hline & & & & $\pm 0,577$ \\
\hline $1 \times /$ dia & 2 & $4,9 \%$ & & \\
\hline $2 x / d i a$ & 2 & $4,9 \%$ & - & \\
\hline Enalapril $20 \mathrm{mg}$ & & & & - \\
\hline $2 x / d i a$ & 1 & $2,4 \%$ & & \\
\hline Losartana $50 \mathrm{mg}$ & & & & $\pm 0,507$ \\
\hline 1x/dia & 6 & $14,6 \%$ & & \\
\hline $2 x /$ dia & 9 & $22 \%$ & - & \\
\hline Losartana $100 \mathrm{mg}$ & & & & - \\
\hline $1 \times$ dia & 1 & $2,4 \%$ & & \\
\hline Insulina NAH & & & & \\
\hline 1x/dia & 1 & $2,4 \%$ & - & - \\
\hline $\begin{array}{l}\text { Andolipino } \\
\text { Cloridrato de benazepril }\end{array}$ & 1 & $2,4 \%$ & - & - \\
\hline $1 x /$ dia & 1 & $2,4 \%$ & - & - \\
\hline Sinvastatina $20 \mathrm{mg}$ & & & & \\
\hline $1 \times /$ dia & 2 & $4,9 \%$ & - & - \\
\hline $\begin{array}{l}\text { Moduretic } \\
\text { Corus }\end{array}$ & 1 & $2,4 \%$ & - & - \\
\hline $\begin{array}{l}\text { 1x/dia } \\
\text { Brasart HCT }\end{array}$ & 1 & $2,4 \%$ & - & - \\
\hline $\begin{array}{l}\text { 1x/dia } \\
\text { Glimepirida } 2 \mathrm{mg}\end{array}$ & 2 & $4,9 \%$ & - & - \\
\hline $\begin{array}{l}\text { 1x/dia } \\
\text { Anqipress }\end{array}$ & 3 & $7,3 \%$ & - & - \\
\hline 1x/dia & 1 & $2,4 \%$ & - & - \\
\hline Captopril 25mg & & & & $\pm 0,378$ \\
\hline $1 \mathrm{cp}$ & 1 & $2,4 \%$ & - & \\
\hline $\begin{array}{l}2 \mathrm{cp} \\
\text { Vasopril 10mg }\end{array}$ & 6 & $14,6 \%$ & & \\
\hline $\begin{array}{l}1 \mathrm{x} / \text { dia } \\
\text { Propranolol } 40 \mathrm{ma}\end{array}$ & 1 & $2,4 \%$ & - & - \\
\hline $\begin{array}{l}2 \mathrm{cp} \\
\text { Capilarema } 75 \mathrm{mg}\end{array}$ & 1 & $2,4 \%$ & - & - \\
\hline $\begin{array}{l}\text { 1x/dia } \\
\text { Ass 100mg }\end{array}$ & 1 & $2,4 \%$ & - & - \\
\hline $1 \mathrm{x} / \mathrm{dia}$ & 1 & $2,4 \%$ & - & - \\
\hline $\begin{array}{l}\text { Corvedilol } 125 \mathrm{mg} \\
3 \mathrm{cp}-3 \times / \text { dia } \\
\text { Furosemida } 40 \mathrm{mg}\end{array}$ & 1 & $2,4 \%$ & - & - \\
\hline $\begin{array}{l}\text { 2x/dia } \\
\text { Bravan } 80 \mathrm{mg}\end{array}$ & 1 & $2,4 \%$ & - & - \\
\hline $1 \times /$ dia & 1 & $2,4 \%$ & - & - \\
\hline
\end{tabular}

Fonte: Dados da pesquisa, 2018.

O tratamento da HAS objetiva a redução da morbimortalidade, demonstrando efetividade 0 uso de diuréticos, inibidores da enzima conversora de angiotensina e bloqueadores dos receptores AT1 da angiotensina II. Para além do uso de medicamentos, os portadores de HAS devem ser orientados quanto ao uso contínuo destes e das eventuais necessidades de ajuste das doses ou substituição de medicamentos frente ao aparecimento de efeitos adversos (MALACHIAS AR, et al., 2016).

Verificou-se que os exames de Hemoglobina Glicosilada (58,5\%), o de Creatinina Sérica $(51,2 \%)$ e o de Microalbuminúrica $(90,2 \%)$ tiveram predominância de respostas negativas, indicando que estes exames não foram realizados, certificado pela porcentagem retratada em parênteses e, de modo detalhado, na Tabela 5. 
Em contrapartida, os exames de Colesterol Total (82,9\%), o de ECG $(92,7 \%)$ e o de Triglicérides (87\%), apresentaram prevalência de respostas positivas e indicativas da realização dos exames. Foram, por fim, analisados os exames realizados pelos pacientes, de modo que se tem na tabela 5 uma descrição detalhada.

Tabela 5 - Exames realizados pelos portadores de HAS cadastrados no HIPERDIA. Cajazeiras - PB.

\begin{tabular}{lcc}
\hline \multicolumn{1}{c}{ Variáveis } & Frequência & Porcentagem (\%) \\
\hline Hemoglobina Glicosilada & & \\
Sim & 17 & $41,5 \%$ \\
Não & 24 & $58,5 \%$ \\
Creatinina Sérica & 20 & $48,8 \%$ \\
Sim & 21 & $51,2 \%$ \\
Não & & $82,9 \%$ \\
Colesterol Total & 34 & $17,1 \%$ \\
Sim & 7 & $92,7 \%$ \\
Não & 38 & $7,3 \%$ \\
ECG & 3 & $78 \%$ \\
Sim & 32 & $22 \%$ \\
Não & 9 & $9,8 \%$ \\
Triglicérides & & $90,2 \%$ \\
Sim & 4 & \\
Não & 37 & \\
Microalbuminúrica & & \\
Sim & & \\
Não & & \\
\hline
\end{tabular}

Fonte: Dados da pesquisa, 2018.

Ainda de acordo com a VII Diretriz Brasileira de Hipertensão Arterial (MALACHIAS AR, et al., 2016) são exames de rotina para o paciente hipertenso: análise de urina, potássio plasmático, glicemia de jejum e $\mathrm{HbA1c}$, ritmo de filtração glomerular estimado, creatinina plasmática, colesterol total, HDL e triglicérides plasmáticos, ácido úrico plasmático, eletrocardiograma convencional. Para acompanhamento de pacientes com suspeita de comprometimento cardíaco é indicado ainda a radiografia de tórax, para as suspeitas clínicas de insuficiência cardíaca o ecocardiograma, em pacientes hipertensos e diabéticos a albuminúria, na presença de sopros carotídeos o US das carótidas, em pacientes com massas abdominais ou sopro abdominal um US renal ou com Dopller, nas suspeitas de doença arterial cononariana o teste ergométrico, e nos pacientes com demência ou distúrbios cognitivos solicita-se a ressonância do cérebro. Nota-se, portanto, que são realizados na Atenção Básica apenas os exames mais simples, não sendo disponibilizados exames mais complexos e necessários ao diagnóstico de complicações ligadas a HAS. A indisponibilidade ocorre devido ao déficit assistencial ainda existente na região, que conta com insuficiente suporte diagnóstico.

\section{CONCLUSÃO}

Constatou-se uma maior incidência de indivíduos acompanhados do sexo feminino, em sua maioria casados, com baixo índice de escolaridade e aposentados. Ainda com base na maioria, tem-se indivíduos com uma autopercepção de serem saudáveis mesmo não praticando atividades físicas, mas que possuem bons hábitos alimentares. Demonstram elevado nível de estresse, e não utilizam substâncias tóxicas, como o álcool e o cigarro. Quando aos dados complicadores, observou-se que a maioria tem 1 ou mais antecedentes familiares, bem como estão acima do peso e considerável amostragem tem diabetes associada. Assim, dentre os fatores que podem influenciar na adesão ao tratamento observa-se a baixa escolaridade, bem como uma autopercepção de uma condição de vida saudável, quanto aos riscos cardiovasculares, temse a associação do diabetes como um complicador da situação de saúde. Observou-se um elevado índice de medicalização e déficits consideráveis quanto a realização dos exames laboratoriais, assim, para que seja possível a atenção integral aos portadores de HAS. 


\section{REFERÊNCIAS}

1. AZEVEDO ECC, et al. Consumo alimentar de risco e proteção para as doenças crônicas não transmissíveis e sua associação com a gordura corporal: um estudo com funcionários da área de saúde de uma universidade pública de Recife (PE), Brasil. Ciência saúde coletiva, 2014; 19(5): 1613-1622.

2. BENSENOR IM, et al. Prevalência de acidente vascular cerebral e de incapacidade associada no Brasil: Pesquisa Nacional de Saúde - 2013. Arquivos de Neuro-Psiquiatria, São Paulo, 2015; 73(9): p. 746-750.

3. BEZERRA ALA, et al. Perfil epidemiologico de idosos hipertensos no Brasil: uma revisão integrativa. Revista de Medicina, 2018; 97(1): p.103.

4. BRASIL MS. - Portaria no 235/GM, de 20/02/2001. Estabelece as diretrizes para a reorganização da atenção aos segmentos populacionais expostos e portadores de hipertensão arterial e de diabetes mellitus.

5. BRASIL MS. Conselho Nacional de Saúde. Comissão Nacional de Ética em Pesquisa. Manual operacional para comitês de ética em pesquisa/ Ministério da Saúde, Conselho Nacional de Saúde, NORMA OPERACIONAL № 001/2013. Brasília: Ministério da Saúde, 2013.

6. BRASIL MS. Portaria 371/GM, de 4 de março de 2002. Diário Oficial da República Federativa do Brasil, Brasília, DF, 2002; 44(S.1): p. 88.

7. CARRAPATO JFL, et al. Programa Mais Médicos: percepção dos usuários e dos profissionais do SUS. Revista Em Pauta, v. 14, n. 38, 2017.

8. DUTRA DD, et al. Cardiovascular disease and associated factors in adults and elderly registered in a basic health unit. J Res Fundam Care Online, 2016; 8(2): p.4501-9.

9. FIRMO JOA, et al. Controle da hipertensão arterial entre adultos mais velhos: ELSI-Brasil. Rev. Saúde Pública, São Paulo, 2018; 52 (supl. 2).

10. GIL AC. Como elaborar projetos de pesquisa. 4. ed. São Paulo: Atlas, 2009.

11. GUERRA DR, et al. Diagnósticos de enfermagem em pacientes com acidente vascular cerebral: revisão integrativa. Revista Brasileira de Enfermagem, 2016; 69(4): p.785-792.

12. HORTENCIO MNS, et al. Efeitos de exercícios físicos sobre fatores de risco cardiovascular em idosos hipertensos. Rev Bras Promoç Saúde, 2018; 31(2): p.1-9.

13. JODAS EMMG, et al. Effects of physical training and potassium supplementation on blood pressure, glucose metabolism and albuminuria of spontaneously hypertensive rats. Jornal Brasileiro de Nefrologia, 2014; 36(3): p. 271-279.

14. MACHADO JC, et al. Análise de três estratégias de educação em saúde para portadores de hipertensão arterial. Ciência saúde coletiva, 2016; 21(2): pp.611-620.

15. MALACHIAS AR, et al. 7 7 Diretriz Brasileira de Hipertensão Arterial. Arquivos Brasileiros de Cardiologia, 2016; 107(3).

16. MARCONI MA, LAKATOS EM. Fundamentos de metodologia cientíica. São Paulo: Atlas, 2010.

17. MOTTER RF, et al. Conhecimento sobre a farmacoterapia por portadores de Hipertensão Arterial Sistêmica. Ciência \& Saúde Coletiva, 2013; 18 (8): pp.2263-2274.

18. MURARO AP, et al. Fatores associados à Hipertensão Arterial Sistêmica autorreferida segundo VIGITEL nas 26 capitais brasileiras e no Distrito Federal em 2008. Ciência \& Saúde Coletiva, 2013; 18(5): pp.1387-1398.

19. NOBRE F, et al. $6^{a}$ Diretrizes de Monitorização Ambulatorial da Pressão Arterial e $4^{\mathrm{a}}$ - Diretrizes de Monitorização Residencial da Pressão Arterial. Arq Bras Cardiol, 2018; 110(5): p.1-29.

20. OLIVEIRA PTGO, ALMEIDA JM. Grupo educativo: análise na mudança do estilo de vida de portadores de hipertensão arterial sistêmica e diabetes tipo 2. Rev Fac Ciênc Méd Sorocaba, 2018; 20(3): p.142-9.

21. PAULA PAB, et al. O uso do medicamento na percepção do usuário do Programa Hiperdia. Ciência \& Saúde Coletiva, 2011; 16(5): p.:2623-2633.

22. SILVA JP, et al. A hipertensão arterial na mídia impressa: análise da revista Veja. Psicologia e Saber Social, 2014; 2(2): p. 191-203.

23. SILVEIRA EL, et al. Prevalência e distribuição de fatores de risco cardiovascular em portadores de doença arterial coronariana no Norte do Brasil. Rev Fac Cienc Med Sorocaba, 2018; 20(3):167-173.

24. SOUZA MFM, et al. Transição da saúde e da doença no Brasil e nas Unidades Federadas durante os 30 anos do Sistema Único de Saúde. Ciênc. saúde coletiva, Rio de Janeiro, 2018; 23(6): p. 1737-1750.

25. TANCRESI FB, et al. Planejamento em saúde. Saúde \& Cidadania. Universidade de São Paulo. Faculdade de Saúde Pública, 2002.

26. WILLIAMS B, et al. Guidelines for the management of arterial hypertension. European Heart Journal, 2018; 39(33): 3121-3104. 